\title{
AN INDEFINITE RANGE INCLUSION THEOREM FOR TRIPLETS OF BOUNDED LINEAR OPERATORS ON A HILBERT SPACE
}

\author{
Michio Seto And Atsushi Uchiyama
}

Abstract. We study triplets of Hilbert space operators satisfying a certain inequality. A range inclusion theorem with norm estimate for those triplets is given with the language of Kreйn space geometry and de Branges-Rovnyak space theory.

Mathematics subject classification (2010): 47B50, 47B32.

Keywords and phrases: Krein space, de Branges-Rovnyak space, Toeplitz operator.

\section{REFERENCES}

[1] J. Agler And J. E. MCCarthy, Pick interpolation and Hilbert function spaces, Graduate Studies in Mathematics, 44. American Mathematical Society, Providence, RI, 2002.

[2] T. ANDO, de Branges spaces and analytic operator functions, Lecture notes, Sapporo, Japan, 1990.

[3] J. A. BALl AND J. W. Helton, A Beurling-Lax theorem for the Lie group $\mathrm{U}(m, n)$ which contains most classical interpolation theory, J. Operator Theory 9 (1983), no. 1, 107-142.

[4] J. A. BALl AND J. W. Helton, Beurling-Lax representations using classical Lie groups with many applications. II, $\operatorname{GL}(n, \mathbb{C})$ and Wiener-Hopf factorization, Integral Equations Operator Theory 7 (1984), no. 3, 291-309.

[5] J. A. Ball and J. W. Helton, Beurling-Lax representations using classical Lie groups with many applications. III, Groups preserving two bilinear forms, Amer. J. Math. 108 (1986), no. 1, 95-174.

[6] J. A. Ball And J. W. Helton, Beurling-Lax representations using classical Lie groups with many applications. IV, $\mathrm{GL}(n, \mathbb{R}), \mathrm{U}^{*}(2 n), \mathrm{SL}(n, \mathbb{C})$, and a solvable group, J. Funct. Anal. 69 (1986), no. 2, 178-206.

[7] M. A. DRITSChel AND J. RovNyAK, Operators on indefinite inner product spaces, Lectures on operator theory and its applications (Waterloo, ON, 1994), 141-232, Fields Inst. Monogr., 3, Amer. Math. Soc., Providence, RI, 1996.

[8] P. A. Fillmore And J. P. Williams, On operator ranges, Advances in Math. 7 (1971), 254-281.

[9] J. W. Helton, J. A. Ball, C. R. Johnson and J. N. Palmer, Operator theory, analytic functions, matrices, and electrical engineering, CBMS Regional Conference Series in Mathematics, 68. Published for the Conference Board of the Mathematical Sciences, Washington, DC; by the American Mathematical Society, Providence, RI, 1987.

[10] Y. Wu, M. SETO AND R. YANG, Krĕn space representation and Lorentz groups of analytic Hilbert modules, Sci. China Math. 61 (2018), no. 4, 745-768. 